\title{
Structural Determination and Bonding Properties of Gas-Phase Ternary [O, Ta, $2 \mathrm{Cl}^{-}$Anion
}

\author{
Sheng-Jie $\mathrm{Lu}^{1,3,{ }^{*}}$ and Kang Wang ${ }^{2 *}$
}

${ }^{1}$ College of Chemistry and Chemical Engineering, Heze University, Heze, Shandong 274015, China ${ }^{2}$ College of Physics and Electronic Engineering, Heze University, Heze, Shandong Province, 274015, China

${ }^{3}$ Beijing National Laboratory for Molecular Sciences, Beijing, 100190, China

*Corresponding authors. E-mail: lushengjie@iccas.ac.cn and wangkang@hezeu.edu.cn 


\section{Experimental and Theoretical methods}

\section{Experimental methods}

The experiments were carried out with a home-built magnetic-bottle photoelectron spectrometer in combination with a laser vaporization cluster source and a linear time-of-flight mass spectrometer, details of which have been published elsewhere ${ }^{1} . \mathrm{OTaC}_{2}{ }^{-}$was obtained by focusing $532 \mathrm{~nm}$ laser light on a rotating and translating Ta target (13 mm diameter). Meanwhile, helium gas ( $\sim 0.4 \mathrm{MPa})$ collided with the laser-produced plasma to form cluster beams. The $\mathrm{C}$ came from the impurities in the carrier gas and the surface of Ta target were sufficient to produce $\mathrm{OTaC}_{2}{ }^{-}$. OTaC ${ }_{2}^{-}$was size-selected, decelerated, and photodetached by $266 \mathrm{~nm}$ laser light. The photoelectrons were energy-analyzed to get its the photoelectron spectrum, calibrated using the photoelectron spectra of $\mathrm{Cu}^{-}$and $\mathrm{Au}^{-}$. The resolution of the photoelectron spectrometer was about $40 \mathrm{meV}$ at the electron kinetic energy of $1 \mathrm{eV}$.

\section{Theoretical methods}

All calculations were conducted using Gaussian $16 .^{2}$ The guess structures of $\mathrm{OTaC}_{2}{ }^{-}$were searched using crystal structure analysis using particle swarm optimization (CALYPSO) software ${ }^{3}$ at the B3LYP4-7/C/O/3-21G/Ta/LanL2DZ level. Structural optimizations and frequency analyses were carried out at B3LYP/C/O/aug-cc-pVTZ $/$ Ta/aug-cc-pVQZ-PP ${ }^{9}$ level. In addition, the HSE06 ${ }^{10}$ and $\mathrm{PBE}^{11}$ functionals are also used for comparing calculations with B3LYP functional. These results obtained from B3LYP, HSE06, and PBE functionals are listed in Table S1 of SI, suggesting that the calculated results at the B3LYP level are closer to the experimental values than those at the HSE06 and PBE levels, as a result, the results from the B3LYP functional are only shown in this work. The single-point energies are calculated at the $\operatorname{CCSD}(\mathrm{T})^{12-13 / C / O / a u g-c c-p V T Z / T a / a u g-c c-p V Q Z-P P ~ l e v e l . ~ T h e ~ c a l c u l a t e d ~ d e t a i l s ~ o f ~ t h e o r e t i c a l ~ v e r t i c a l ~}$ detachment energies (VDEs) and adiabatic detachment energies (ADEs) can be found in our previous work. ${ }^{14}$ Natural population analysis (NPA) ${ }^{15-17}$ at the B3LYP level was used to give insight into the charge distributions.

(1)H. G. Xu; Z. G. Zhang; Y. Feng; J. Y. Yuan; Y. C. Zhao; W. J. Zheng, Vanadium-Doped Small Silicon Clusters: Photoelectron Spectroscopy and Density-Functional Calculations. Chem. Phys. Lett. 2010, 487, 204-208.

(2)Gaussian 16, Revision C.01, M. J. Frisch, G. W. Trucks, H. B. Schlegel, G. E. Scuseria, M. A. Robb, J. R. Cheeseman, G. Scalmani, V. Barone, G. A. Petersson, H. Nakatsuji, X. Li, M. Caricato, A. V. Marenich, J. Bloino, B. G. Janesko, R. Gomperts, B. Mennucci, H. P. Hratchian, J. V. Ortiz, A. F. Izmaylov, J. L. Sonnenberg, D. Williams-Young, F. Ding, F. Lipparini, F. Egidi, J. Goings, B. Peng, A. Petrone, T. Henderson, D. Ranasinghe, V. G. Zakrzewski, J. Gao, N. Rega, G. Zheng, W. Liang, M. Hada, M. Ehara, K. Toyota, R. Fukuda, J. Hasegawa, M. Ishida, T. Nakajima, Y. Honda, O. Kitao, H. Nakai, T. Vreven, K. Throssell, J. A. Montgomery, Jr., J. E. Peralta, F. Ogliaro, M. J. Bearpark, J. J. Heyd, E. N. Brothers, K. N. Kudin, V. N. Staroverov, T. A. Keith, R. Kobayashi, J. Normand, K. Raghavachari, A. P. Rendell, J. C. Burant, S. S. Iyengar, J. Tomasi, M. Cossi, J. M. Millam, M. Klene, C. Adamo, R. Cammi, J. W. Ochterski, R. L. Martin, K. Morokuma, O. Farkas, J. B. Foresman, and D. J. Fox, Gaussian, Inc., Wallingford CT, 2016.

(3)J. Lv; Y. C. Wang; L. Zhu; Y. M. Ma, Particle-Swarm Structure Prediction on Clusters. J. Chem. Phys. 2012, $137,084104$. 
(4)A. D. Becke, Density-Functional Exchange-Energy Approximation with Correct Asymptotic Behavior. Phys. Rev. A 1988, 38, 3098-3100.

(5)C. Lee; W. Yang; R. G. Parr, Development of the Colle-Salvetti Correlation-Energy Formula into a Functional of the Electron Density. Phys. Rev. B: Condens. Matter Mater. Phys. 1988, 37, 785-789.

(6)A. D. Becke, Density-Functional Thermochemistry. Iii. The Role of Exact Exchange. J. Chem. Phys. 1993, 98, 5648-5652.

(7)P. J. Stephens; F. J. Devlin; C. F. Chabalowski; M. J. Frisch, Ab Initio Calculation of Vibrational Absorption and Circular Dichroism Spectra Using Density Functional Force Fields. J. Phys. Chem. 1994, 98, 11623-11627.

(8)D. E. Woon; T. H. Dunning, Gaussian Basis Sets for Use in Correlated Molecular Calculations. Iii. The Atoms Aluminum through Argon. J. Chem. Phys. 1993, 98, 1358-1371.

(9)K. A. Peterson; D. Figgen; M. Dolg; H. Stoll, Energy-Consistent Relativistic Pseudopotentials and Correlation Consistent Basis Sets for the 4d Elements Y-Pd J. Chem. Phys. 2007, 126, 124101.

(10)A. V. Krukau; O. A. Vydrov; A. F. Izmaylov; G. E. Scuseria, Influence of the Exchange Screening Parameter on the Performance of Screened Hybrid Functionals J. Chem. Phys. 2006, 125, 224106.

(11)J. P. Perdew; K. Burke; M. Ernzerhof, Generalized Gradient Approximation Made Simple. Phys. Rev. Lett. 1996, 77, 3865-3868.

(12)G. D. Purvis; R. J. Bartlett, A Full Coupled-Cluster Singles and Doubles Model: The Inclusion of Disconnected Triples. $J$. Chem. Phys. 1982, 76, 1910-1918.

(13)G. E. Scuseria; H. F. Schaefer, Is Coupled Cluster Singles and Doubles (CCSD) More Computationally Intensive Than Quadratic Configuration Interaction (Qcisd)? J. Chem. Phys. 1989, 90, 3700-3703.

(14)S.-J. Lu; H.-G. Xu; X.-L. Xu; W.-J. Zheng, Structural Evolution and Electronic Properties of $\mathrm{TaSi}_{\mathrm{n}}{ }^{-10}(\mathrm{n}=2-15)$ Clusters: Size-Selected Anion Photoelectron Spectroscopy and Theoretical Calculations. J. Phys. Chem. A 2020, 124, 9818-9831.

(15)J. E. Carpenter. Extension of Lewis Structure Concepts to Open-Shell and Excited-State Molecular Species. PhD. Thesis, University of Wisconsin, Madison, WI, 1987.

(16)J. E. Carpenter; F. Weinhold, Analysis of the Geometry of the Hydroxymethyl Radical by the "Different Hybrids for Different Spins" Natural Bond Orbital Procedure. J. Mol. Struct. : THEOCHEM 1988, 169, 41-62.

(17)A. E. Reed ; L. A. Curtiss; F. Weinhold, Intermolecular Interactions from a Natural Bond Orbital, Donor-Acceptor Viewpoint. Chem. Rev. 1988, 88, 899-926. 


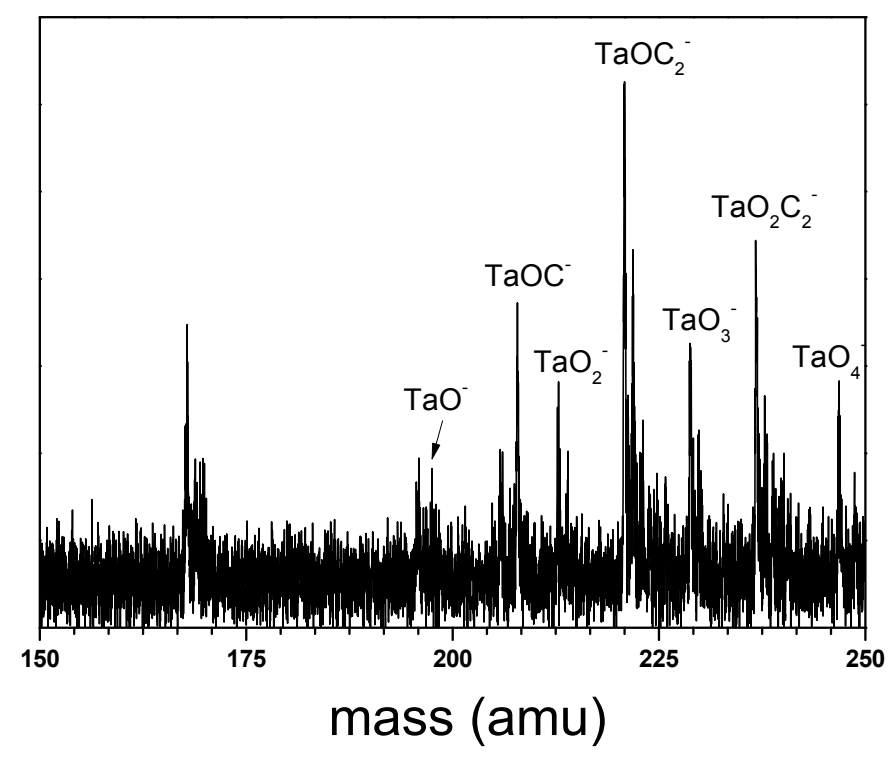

Figure S1. Mass spectrum of anions. 
Table S1. Relative energies, theoretical VDEs and ADEs of the low-energy isomers of $\mathrm{OTaC}_{2}{ }^{-}$, along with the experimental VDEs and ADEs. The isomer labeled in bold are the most probable isomers in the experiments. The relative energies, and theoretical VDEs and ADEs are calculated at the B3LYP, HSE06, and PBE levels. The uncertainties of experimental VDE and ADE are $\pm 0.08 \mathrm{eV}$.

\begin{tabular}{|c|c|c|c|c|c|c|c|c|c|c|c|c|c|}
\hline \multirow[b]{2}{*}{ Isomers } & \multirow[b]{2}{*}{$\begin{array}{c}\text { Electronic } \\
\text { state }\end{array}$} & \multicolumn{4}{|c|}{$\Delta \mathrm{E}(\mathrm{eV})$} & \multicolumn{4}{|c|}{ VDE (eV) } & \multicolumn{4}{|c|}{$\overline{\mathrm{ADE}}(\mathrm{eV})$} \\
\hline & & $\operatorname{CCSD}(\mathrm{T})$ & B3LYP & HSE06 & PBE & $\begin{array}{l}\text { Theo. } \\
\text { B3LYP }\end{array}$ & $\begin{array}{c}\text { Theo. } \\
\text { HSE06 }\end{array}$ & $\begin{array}{l}\text { Theo. } \\
\text { PBE }\end{array}$ & Expt. & $\begin{array}{c}\text { Theo. } \\
\text { B3LY } \\
\text { P }\end{array}$ & $\begin{array}{c}\text { Theo. } \\
\text { HSE06 }\end{array}$ & $\begin{array}{r}\text { Theo. } \\
\text { PBE }\end{array}$ & Expt. \\
\hline $112 A$ & ${ }^{1} \mathrm{~A}^{\prime}$ & 0.00 & 0.00 & 0.00 & 0.00 & 2.73 & 2.46 & 2.39 & 2.85 & 2.56 & 2.40 & 2.33 & 2.58 \\
\hline $112 \mathrm{~B}$ & ${ }^{1} \mathrm{~A}^{\prime}$ & 0.86 & 0.90 & 0.83 & 0.62 & 1.49 & 1.36 & 1.43 & & 1.39 & 1.31 & 1.37 & \\
\hline $112 \mathrm{C}$ & ${ }^{1} \Sigma^{+}$ & 1.56 & 2.22 & 205 & 1.90 & 1.43 & 1.39 & 1.58 & & 0.78 & 0.84 & 1.08 & \\
\hline
\end{tabular}


Table S2. Cartesian coordinates of the low-lying isomers of $\mathrm{OTaC}_{2}{ }^{-/ 0}$.

\begin{tabular}{|c|c|c|c|c|c|c|c|}
\hline \multicolumn{4}{|c|}{$112 \mathrm{~A}$} & \multicolumn{4}{|c|}{$112 \mathrm{~B}$} \\
\hline & $\mathrm{X}$ & Y & Z & & $\mathrm{X}$ & $\mathrm{Y}$ & Z \\
\hline $\mathrm{O}$ & 0.81238400 & 1.42755800 & 0.00015700 & $\mathrm{C}$ & -1.70685300 & 0.46709200 & -0.65130700 \\
\hline $\mathrm{C}$ & -2.09427500 & 0.82520600 & -0.00025700 & $\mathrm{O}$ & 1.38510900 & 1.02378600 & 0.00000300 \\
\hline $\mathrm{C}$ & -1.75622100 & -0.40457100 & 0.00036300 & $\mathrm{C}$ & -1.70675500 & 0.46719100 & 0.65146900 \\
\hline $\mathrm{Ta}$ & 0.22745100 & $\begin{array}{l}-0.19101800 \\
112 \mathrm{C}\end{array}$ & -0.00002600 & $\mathrm{Ta}$ & 0.12877800 & -0.18898600 & -0.00001400 \\
\hline & $\mathrm{X}$ & Y & Z & & & & \\
\hline $\mathrm{C}$ & -1.65441200 & 0.09037800 & 0.00002100 & & & & \\
\hline $\mathrm{O}$ & -2.74869600 & -0.42860100 & 0.00000100 & & & & \\
\hline $\mathrm{C}$ & -0.85858700 & 1.21897000 & 0.00001900 & & & & \\
\hline $\mathrm{Ta}$ & 0.50777500 & -0.06064800 & -0.00000300 & & & & \\
\hline & & $112 A^{\prime}$ & & & & $112 \mathrm{~B}^{\prime}$ & \\
\hline & $\mathrm{X}$ & Y & Z & & $\mathrm{X}$ & $\mathrm{Y}$ & Z \\
\hline $\mathrm{C}$ & 1.52833800 & 0.71817200 & 0.52574100 & $\mathrm{O}$ & -0.67223200 & 1.45569200 & 0.00016600 \\
\hline $\mathrm{O}$ & -1.32902100 & 1.02493600 & -0.16686100 & $\mathrm{C}$ & 2.00170300 & 0.78714300 & 0.00021400 \\
\hline $\mathrm{C}$ & 1.98652100 & 0.19719300 & -0.53650700 & $\mathrm{C}$ & 1.70452600 & -0.46548200 & 0.00004800 \\
\hline $\mathrm{Ta}$ & -0.14324600 & $\begin{array}{l}-0.18755700 \\
112 C^{\prime}\end{array}$ & 0.01917100 & $\mathrm{Ta}$ & -0.23095200 & -0.18596600 & -0.00004000 \\
\hline & $\mathrm{X}$ & $\mathrm{Y}$ & Z & & & & \\
\hline $\mathrm{O}$ & -0.00367700 & -3.61592700 & 0.00000000 & & & & \\
\hline$C$ & 0.00042100 & -2.45476600 & 0.00000000 & & & & \\
\hline $\mathrm{C}$ & 0.00448100 & -1.15833900 & 0.00000000 & & & & \\
\hline Тa & 0.00000000 & 0.69323300 & 0.00000000 & & & & \\
\hline
\end{tabular}

